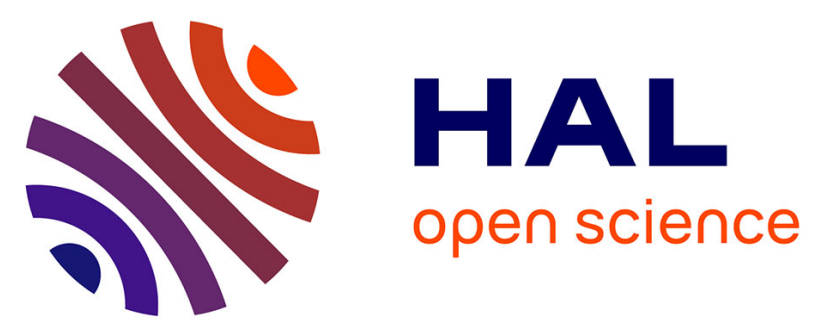

\title{
Dynamic modeling highlights the major impact of droplet coalescence on the in vitro digestion kinetics of a whey protein stabilized submicron emulsion
}

Thuy Minh Giang, Steven Le Feunteun, Sebastien Gaucel, Pierre Brestaz, Marc Anton, Anne Meynier, Ioan-Cristian Trelea

\section{To cite this version:}

Thuy Minh Giang, Steven Le Feunteun, Sebastien Gaucel, Pierre Brestaz, Marc Anton, et al.. Dynamic modeling highlights the major impact of droplet coalescence on the in vitro digestion kinetics of a whey protein stabilized submicron emulsion. Food Hydrocolloids, 2015, 43, pp.66-72. 10.1016/j.foodhyd.2014.04.037 . hal-01195524

\section{HAL Id: hal-01195524 \\ https://hal.science/hal-01195524}

Submitted on 11 Jul 2017

HAL is a multi-disciplinary open access archive for the deposit and dissemination of scientific research documents, whether they are published or not. The documents may come from teaching and research institutions in France or abroad, or from public or private research centers.
L'archive ouverte pluridisciplinaire HAL, est destinée au dépôt et à la diffusion de documents scientifiques de niveau recherche, publiés ou non, émanant des établissements d'enseignement et de recherche français ou étrangers, des laboratoires publics ou privés. 
1 Dynamic modeling highlights the major impact of droplet coalescence on

2 the in vitro digestion kinetics of a whey protein stabilized submicron

\section{emulsion}

4

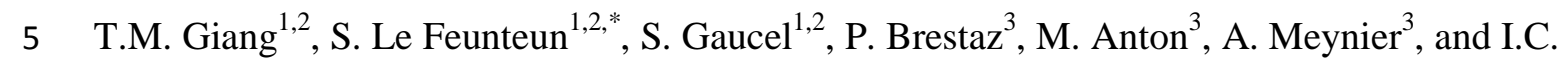

7

$8{ }^{1}$ INRA, UMR782 Génie et Microbiologie des Procédés Alimentaires, F-78850 Thiverval

9 Grignon, France

$10{ }^{2}$ AgroParisTech, UMR782 Génie et Microbiologie des Procédés Alimentaires, F-78850

11 Thiverval Grignon, France

$12{ }^{3}$ INRA, UR1268 Biopolymères Interactions Assemblages, F-44300 Nantes, France

13

14 *Corresponding author: steven.le-feunteun@grignon.inra.fr, +33(0)13814596 


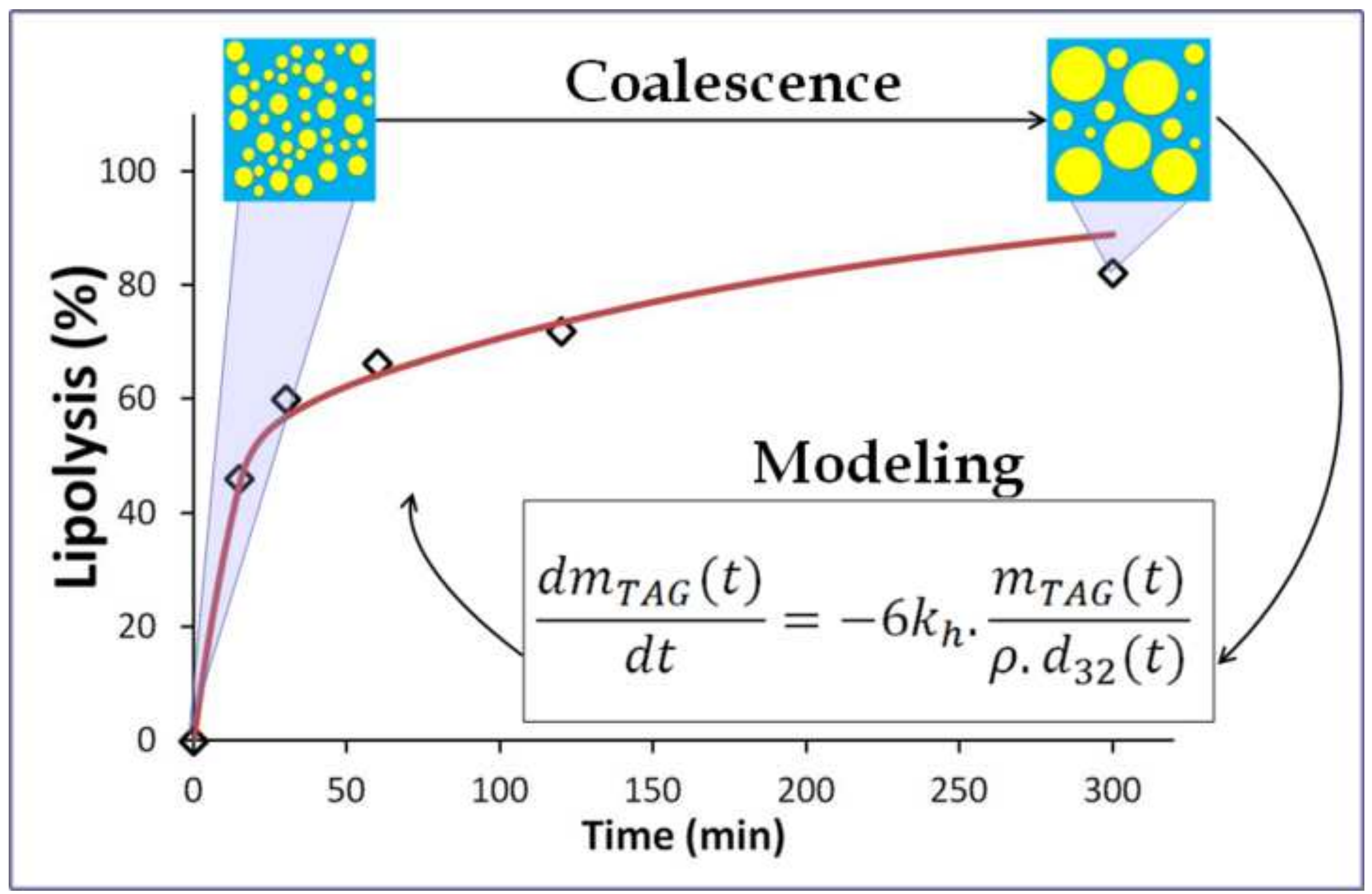




\section{Highlights:}

- A whey protein stabilized emulsion was submitted to in vitro digestion.

- The kinetics of pancreatic lipolysis plateaued after $~ 30$ min of intestinal digestion.

- A marked coalescence of the oil droplets occurred concurrently.

- A mathematical model was developed and successfully used to relate both sets of data.

- Droplet coalescence, and not enzyme inhibition, was the key mechanism explaining the rate of lipid digestion. 


\section{Abstract}

16 Whey protein stabilized submicron oil-in-water emulsions have been reported to remain

17 relatively stable in size during the gastric phase and to coalesce during the intestinal phase of

18 in vitro digestion experiments. The aim of this study was to understand the impact of oil

19 droplet coalescence on the intestinal lipolysis kinetics during an in vitro digestion of such

20 emulsion, and to develop a mathematical model able to predict the experimental observations.

21 A submicron whey protein stabilized emulsion made of a mixture of medium-chain (MCT)

22 and long-chain triacylglycerols (LCT) was prepared and submitted to gastro-intestinal in vitro

23 digestion. Triacylglycerol concentrations and droplet size distributions were measured before

24 and after the gastric phase and during the intestinal phase using HPLC and laser granulometry,

25 respectively. MCT were fully digested within 15 min of intestinal digestion, whereas LCT

26 were still detected after 5 hours. Moreover, the intestinal lipolysis of LCT showed a two-stage

27 behavior with an initial fast rate that markedly slowed down after about $30 \mathrm{~min}$, a time at

28 which a sudden rise in the droplet sizes, attributed to coalescence, was also observed. A

29 mathematical model based on the experimentally measured droplet sizes and assuming a rate

30 of lipolysis proportional to the interfacial area was developed and successfully used to

31 reproduce the observed kinetics. Our results support the idea that droplet coalescence during

32 the intestinal phase was the main reason for the marked slowdown of the kinetics of lipid

33 digestion, hence suggesting that inhibition of the lipolysis reaction could be a secondary

34 factor only.

35

36 Keywords: Pancreatic lipase, Lipid digestion, Droplet size, Polyunsaturated Fatty Acids,

37 Simulation. 
39 Key parameters that govern food digestion in vivo are very hard to identify for practical 40 reasons. In vitro methods, for which the conditions are strictly controlled, have therefore been, 41 and are still, largely used. Concerning the digestion of emulsified lipids, both their 42 compositional and structural properties can affect digestion [1]. It is known that short chain

43 fatty acids are released faster and to greater extents than long-chain fatty acids [2-6], and that 44 polyunsaturated fatty acids such as docosahexanoic acid (DHA) and eicosapentaenoic acid 45 (EPA) are highly resistant to lipolysis [7-8]. The composition and the surface area of the 46 interface surrounding lipids are also very important parameters because digestive lipases must 47 adsorb on the droplet surface to reach their substrates. On the one hand, the kinetics of 48 lipolysis increases with the interfacial area, and hence with decreasing droplet sizes [3, 5-6, 949 10]. On the other hand, the rate of the reaction depends on the lipase affinity for the interfacial 50 layer and may therefore be modulated by the nature of the emulsifiers used to stabilize the 51 emulsions [11-12].

52 Moreover, if lipid digestion is initiated in the stomach by the gastric lipase, most of the 53 reaction $(70-90 \%)$ is performed in the upper part of the intestine by the combined action of 54 the pancreatic lipase, its colipase, and bile salts [1]. Beyond the properties of the native 55 emulsions, the structural modifications they may undergo within the stomach will affect the 56 intestinal phase, and hence the overall kinetics of the digestion [13]. Emulsions can remain 57 stable, flocculate or coalesce during the gastric phase depending on several parameters such 58 as the initial droplet size, the type of emulsifiers, and the composition of the surrounding 59 medium [14-17]. The rate of lipid hydrolysis during the intestinal phase can thus be impacted 60 by changes in the droplet surface area induced by the gastric structuring of the emulsions: the 61 lower the interfacial area when emptied into the duodenum, the slower the digestion. The 
62 stability of the oil droplets against flocculation and coalescence in the stomach is therefore

63 essential to understand why two resembling emulsions may lead to different digestion kinetics.

64 Several studies have also highlighted that some emulsions can remain relatively stable during

65 the gastric phase but undergo important modifications during the intestinal phase of digestion.

66 Whey protein stabilized submicron emulsions are good examples thereof. They have been

67 reported to remain stable in size or flocculate in simulated gastric conditions [14, 18-19]. In

68 either case, the initial rate of lipolysis during the subsequent intestinal phase was fast and

69 comparable to other gastric stable emulsions [14], suggesting that flocculation during the

70 gastric step did not alter much the intestinal lipolysis kinetics. In parallel, other studies have

71 shown that whey protein stabilized emulsions are highly prone to coalescence during the

72 intestinal phase of in vitro digestion [11, 13, 19-20]. The effect of such an intestinal

73 coalescence of the oil droplets on the kinetics of pancreatic lipolysis have, however, not been

74 investigated so far.

75 Therefore, our study aimed at studying and modeling the influence of droplet coalescence on

76 the kinetics of pancreatic lipolysis during an in vitro gastrointestinal digestion of a whey

77 protein stabilized emulsion. The emulsion was prepared from a blend of medium-chain

78 triacylglycerols and a microalgae oil rich in docosahexaenoic acid (DHA), a long-chain $\omega 3$

79 polyunsaturated fatty acid which daily recommended intake is $250 \mathrm{mg}$ [21]. The evolution of

80 both the oil droplet sizes and the lipolysis kinetics were monitored throughout gastro-

81 intestinal in vitro digestion experiments. A mathematical model, in line with previously

82 published ones [5, 22-23], was developed to quantitatively evaluate the relationships between

83 the intestinal lipolysis kinetics and the evolution of the droplet surface area. 
$85 \quad 2.1$ Materials

86 The oil containing medium-chain triacylglycerols (Miglyol 812S) (MCT): C8:0 (54\%) and 87 C10:0 (43\%) was purchased from Sasol GmbH, Germany. The oil containing long-chain 88 triacylglycerols (DHAsco) (LCT): docosahexahenoic acid (DHA, C22:6 n-3, 40\%), C12:0 89 (4\%), C14:0 (12\%), C16:0 (12\%) and C18:1 n-9 (24\%) was obtained from Martek, via DSM

90 Nutritional Products Ldt, Switzerland. Whey protein powder (Prolacta 95) was purchased 91 from Lactalis Ingredients, France. Pepsin (P7012), mucin (M2378), pancreatin (P7545), 92 pancreatic lipase (L3126) and bile extract (B8631) were from porcine origin and obtained 93 from Sigma-Aldrich, France. Water was Milli-Q water. Solvents for liquid chromatography 94 were chloroform for HPLC (Carlo Erba), methyl alcohol for HPLC (99.9\%, Carlo Erba), 95 ammonia solution (30\%, Carlo Erba).

96

\section{2.2. Emulsion preparation}

98 An emulsion composed of $80 \%$ of aqueous phase and $20 \%$ of oil (w/w) was made. The 99 aqueous phase was prepared by dissolving $4 \%$ (w/w) of whey protein powder, used as 100 emulsifier, in a $0.1 \mathrm{M}$ sodium phosphate buffer at $\mathrm{pH} 7.0$. The oil phase consisted of $62.5 \%$

101 LCT and 37.5\% MCT (w/w) mixed together. A rotor-stator homogenizer (SilentCrusher M 102 equipped with the $12 \mathrm{~F}$ generator from Heidolph Instruments, Germany) was used in a pre103 emulsification step (5 min, $20000 \mathrm{rpm}$ ). The coarse emulsion was successively homogenized 104 for 5 min at 500 bars and for 10 min at 1000 bars under nitrogen flow with a high-pressure 105 homogenizer (C3-EmulsiFlex, Sodexim SA, France) temperature-controlled at $4^{\circ} \mathrm{C}$ to produce 106 the emulsion with droplet diameters below micron. A $50 \%(\mathrm{w} / \mathrm{w})$ maltodextrin in water 107 solution was then added as a 1:1 (v/v) ratio to the emulsion and the mixture was thereafter 108 freeze-dried. The dried emulsion was then conditioned in oxygen hermetic bag under vacuum 
109 and kept at $-20^{\circ} \mathrm{C}$ until uses. The dried emulsion was rehydrated in Milli-Q water to obtain a

110 final oil concentration of $3.2 \%(\mathrm{w} / \mathrm{w})$ on the day of the in vitro experiments.

111

\section{$112 \quad 2.3$ Emulsion digestion}

$1133 \mathrm{~mL}$ of the rehydrated emulsion, corresponding to an oil mass of about $96 \mathrm{mg}$, was placed

114 into $22.4 \mathrm{~mL}$ headspace vials hermetically sealed with Teflon/silicon septa and aluminum

115 caps. These vials were kept in a temperature controlled chamber at $37^{\circ} \mathrm{C}$ under magnetic

116 stirring (400 rpm. $\mathrm{min}^{-1}$ ) throughout the duration of the experiments. The gastric phase

117 duration was $60 \mathrm{~min}$ and was launched by adding $2.12 \mathrm{~mL}$ of simulated gastric fluid (SGF)

118 and $40 \mu \mathrm{L}$ of $1 \mathrm{M} \mathrm{HCl}$ to reach a final $\mathrm{pH}$ of 2.5 in the reaction vials. The SGF solution

119 contained 3.9 g.L $\mathrm{L}^{-1}$ of pepsin, 2.4 g.L $\mathrm{L}^{-1}$ of mucin, $120 \mathrm{mM}$ of $\mathrm{NaCl}, 2 \mathrm{mM}$ of $\mathrm{KCl}$ and $6 \mathrm{mM}$

120 of $\mathrm{CaCl}_{2}$. The intestinal phase duration was then launched for 300 min maximum by adding

$1214.86 \mathrm{~mL}$ of simulated intestinal fluid (SIF) into the vials and $100 \mu \mathrm{L}$ of $1 \mathrm{M} \mathrm{NaCO}_{3}$ to reach a

122 final $\mathrm{pH}$ of 6.5 . The simulated intestinal fluid (SIF) contained $30.8 \mathrm{~g} . \mathrm{L}^{-1}$ of bile extract

123 powder, 0.82 g.L. $\mathrm{L}^{-1}$ of pancreatin, $0.41 \mathrm{~g} . \mathrm{L}^{-1}$ of pancreatic lipase, and the same electrolyte

124 concentrations as the SGF.

125 The native emulsion (NE), and samples taken at the end of the gastric phase (G60), at $\mathrm{t}=0$

126 min of the intestinal phase (I0) using a modified SIF that contained all constituents except

127 pancreatin and lipase, and at 15, 30, 60, 120 and 300 min of intestinal digestion (I15 to I300)

128 were analyzed. One vial was used for one sampling time and one type of measurement

129 (quantification of LCT and MCT by HPLC or droplet size by laser granulometry) so that the

130 contents of 16 vials were analyzed in total ( 2 methods times 8 sampling times) for one

131 digestion. Three independent digestion experiments, further denoted replicates, were

132 performed. Samples devoted to droplet size measurements were analyzed immediately,

133 whereas samples devoted to HPLC measurements were kept at $-80^{\circ} \mathrm{C}$ until further analysis. 


\subsection{Quantification of LCT and MCT by HPLC}

136 HPLC paired with an evaporative light scattering detector (ELSD) was used to quantify the 137 decrease of both LCT and MCT masses during the time course of the in vitro digestions. Total 138 lipids were extracted from $1.5 \mathrm{~mL}$ of the native emulsions or of the stomach media and from 3 $139 \mathrm{~mL}$ of the intestinal media according to the Bligh and Dyer [24] procedure with minor 140 modifications in the ratio $\mathrm{CHCl}_{3} / \mathrm{CH}_{3} \mathrm{OH} / \mathrm{H}_{2} \mathrm{O} 1 / 2 / 1$. Before the HPLC analyses, the lipid 141 extract was dissolved in $\mathrm{CHCl}_{3}$ down to $0.3 \mathrm{mg} \cdot \mathrm{mL}^{-1}$ for native emulsion and stomach media 142 and to $0.7 \mathrm{mg} \cdot \mathrm{mL}^{-1}$ for intestinal media. HPLC operating conditions were similar to those 143 described in [25] using a Uptip-prep Strategy column $(2.2 \mu \mathrm{m}$ SI, $150 \times 4.6 \mathrm{~mm}$, Interchim, 144 Montluçon, France) and $30 \mu \mathrm{L}$ of injected lipid extract. As illustrated in Fig. 1, injected 145 masses of pure MCT and LCT ranging from 0.5 to $9 \mu \mathrm{g}$ led to a power law calibration curve 146 with no distinction of the TAG chain-length.

147 The triacylglycerol region of an HPLC chromatogram stemming from an undigested sample is 148 presented in Fig. 2. The retention times of LCT (1.21 min) and MCT (1.32 min) were 149 different but their signals partly overlapped. As illustrated in Fig. 2, signal deconvolution was 150 therefore undertaken using a specifically developed algorithm running with the Matlab ${ }^{\mathrm{TM}}$

151 software (The MathWorks Inc., Natick, MA) and the recovered LCT and MCT signals were 152 converted into masses using the calibration curve (Fig. 1). Reliable results were obtained 153 using this procedure as shown from the comparison of the mean LCT and MCT masses of $15457.3 \pm 2.7$ and $35.0 \pm 2.0 \mathrm{mg}$ (estimated over 15 undigested samples) with the 59.7 and 35.7

155 mg targeted masses in each digestion vial, respectively. LCT and MCT masses were finally 156 converted into lipolysis percentages using Eq. 1:

$$
\operatorname{lipolysis}(t)=\frac{m_{T A G_{0}}-m_{T A G}(t)}{m_{T A G_{0}}} \times 100
$$


157 where $m_{T A G_{0}}$ and $m_{T A G}(t)$ are the masses $(\mathrm{mg})$ of LCT or MCT initially present in the vials 158 and measured by HPLC at time t, respectively.

159

\section{$160 \quad 2.5$ Droplets size measurement}

161 The volume-based distribution of oil droplet sizes was measured using a Mastersizer S 162 (Malvern Instruments Ltd., Worcestershire, UK) equipped with a $2 \mathrm{~mW} \mathrm{He}-\mathrm{Ne}$ laser of $\lambda=$ $163633 \mathrm{~nm}$ and the $300 \mathrm{RF}$ lens with detection limits of 0.05 and $900 \mu \mathrm{m}$. The refractive index $n_{0}$ 164 of the aqueous phase was 1.33 and the properties of the dispersed phase were 1.457 for the 165 refractive index and 0.001 for the absorption. Samples were pre-diluted 100 -fold with the 166 desired solution (with or without sodium dodecyl sulfate, SDS, as deflocculating agent), and 167 then diluted with distilled water to reach an oil volume concentration near $0.01 \%$ for the 168 circulation in the measurement cell. All analyses were performed at room temperature as 169 described previously $[8,25]$. The surface-weighted mean diameter, $d_{32}$, corresponding to the 170 droplet diameter having the same ratio of volume to surface area as the droplet distribution, 171 was calculated according to:

$$
d_{32}=\frac{\sum_{i} n_{i} d_{i}^{3}}{\sum_{i} n_{i} d_{i}^{2}}
$$

172 where $n_{i}$ is the number of droplets of diameter $d_{i}$.

173

174

\section{Mathematical modeling}

\section{$175 \quad 3.1$ Model assumptions and equations}

176 Lipolysis is mediated by the pancreatic colipase-lipase system which absorbs onto the droplet 177 interface and splits the $s n-1$ and $s n-3$ ester bonds of triacylglycerols (TAG). The first step of 178 the reaction generates one free fatty acid (FFA) and a diacylglycerol (DAG) which is further 179 transforms into a second FFA and the sn-2-monoacylglycerol. Our model simulates the 
180 kinetics of lipolysis as inferred from the disappearance of the TAG molecules so that, in

181 principle, it only characterizes the first step of the reaction. Nevertheless, the lack of detected

182 DAG in the course of the experiments strongly suggests that the second reaction step was not

183 rate-limiting in our experimental conditions, and hence that similar lipolysis kinetics would

184 have been recovered by monitoring the appearance of the final products of the reaction. The

185 main modeling assumptions were as follows:

186 A1: The rate of TAG hydrolysis was assumed proportional to the interfacial area of the 187 droplets $[13-14,22]$. This means that the surface reaction rate was taken as constant in the 188 considered experimental conditions. Thus:

$$
\frac{d m_{T A G}(t)}{d t}=-k_{h} \cdot A(t)
$$

189 where $k_{h}$ is the hydrolysis rate constant $\left(\mathrm{mg} \cdot \mathrm{m}^{-2} \cdot \mathrm{min}^{-1}\right)$ and $A(t)$ is the interfacial area $\left(\mathrm{m}^{2}\right)$ at 190 time $\mathrm{t}(\mathrm{min})$.

191 A2: The droplets were considered as spheres, and the interfacial area of the droplets was 192 assumed to be adequately described by the size distributions measured by laser granulometry 193 and their corresponding $d_{32}$. Hence, one can write that:

$$
A(t)=6 \frac{V_{T A G}(t)}{d_{32}(t)}
$$

194 where $d_{32}(t)$ is the surface-weighted mean diameter $(\mathrm{nm})$ at time $\mathrm{t}$, and $V_{T A G}(t)$ is the 195 volume of TAG in the sample $\left(\mathrm{mm}^{3}\right)$ at time t. Eq. 3 can be further transformed into:

$$
A(t)=6 \frac{m_{T A G}(t)}{\rho \cdot d_{32}(t)}
$$

196 where $\rho$ is the mass density of TAG $\left(\mathrm{mg} / \mathrm{mm}^{3}\right)$ and $m_{\text {TAG }}(t)$ is the mass of TAG $(\mathrm{mg})$ at time $197 \mathrm{t}$.

198 Combining Eq. 1 and 4, the evolution of the TAG mass is given by:

$$
\frac{d m_{T A G}(t)}{d t}=-6 k_{h} \cdot \frac{m_{T A G}(t)}{\rho \cdot d_{32}(t)}
$$


199 To solve the differential equation Eq. 5, one also needs to know how the droplet sizes evolve

200 as a function of time. In the present study, we resorted to two different assumptions on $d_{32}(t)$,

201 leading to two different versions of the model:

202 A3a: In one version, it was assumed that all droplets had the same diameter and that the total

203 number of droplets remained constant, hypotheses that lead to the following equation [5]:

$$
d_{32}(t)=d_{0} \sqrt[3]{\frac{m_{T A G}(t)}{m_{T A G_{0}}}}
$$

204 where $m_{T A G_{0}}$ is the mass of TAG initially introduced in the reaction vial and $d_{0}$ is the droplet 205 diameter of the native emulsion.

206 A3b: In the other version, $d_{32}(t)$ was estimated by linear interpolations of the experimental 207 values recovered using laser granulometry.

208 For both model versions, the masses calculated by solving Eq. 5 were finally converted into 209 percentages of lipolysis using Eq. 1 to enable the comparison of the model simulations with 210 experimental data.

\section{3.2 Model fitting and parameter estimation}

213 The lipolysis of MCT was so fast that it was already finished at $\mathrm{t}=15 \mathrm{~min}$, i.e. the first 214 sampling time. Only the lipolysis of LCT was therefore considered to confront the model to 215 the experimental data. The differential equation Eq. 5 was numerically solved using a LCT 216 mass density, $\rho$, of $0.92 \mathrm{~g} . \mathrm{cm}^{-3}$, and each of the previously described assumptions (A3a or b) 217 for the droplet size evolution as a function of time, $d_{32}(t)$. The unknown hydrolysis rate, $k_{h}$, 218 was then estimated by fitting model predictions to the LCT lipolysis results determined by 219 HPLC. The recovered value, expressed in $\mathrm{mg}$ of TAG per minute and square meter of 220 interfacial area $\left(\mathrm{mg} \cdot \mathrm{m}^{-2} \cdot \mathrm{min}^{-1}\right)$, was then converted into $\mu \mathrm{mol} \cdot \mathrm{m}^{-2} \cdot \mathrm{min}^{-1}$ for comparison 
221 purposes with the literature using a LCT molar mass of 900 g.mol ${ }^{-1}$. Numeric calculations

222 were performed using Matlab ${ }^{\mathrm{TM}}$ software (The MathWorks Inc., Natick, MA).

223

224

\section{Results and Discussion}

\section{4.1 Kinetics of lipolysis of LCT and MCT}

226 The evolution of HPLC chromatograms during the intestinal phase stemming from one in

227 vitro digestion experiment is shown in Fig. 3A, and the mean percentages of lipolysis

228 calculated over the three replicates are presented in Fig. 3B. Initially, 2 peaks were clearly

229 visible in the chromatograms. The signal of the native emulsion and at $t=0$ min of the

230 intestinal phase (modified SIF with no lipase) were similar since no gastric lipase was used in

231 this study. At $\mathrm{t}=15 \mathrm{~min}$ of intestinal digestion, the signal arising from MCT entirely

232 disappeared, indicating that MCT were fully hydrolyzed in only few minutes. In contrast,

233 about $20 \%$ of the initial LCT mass was still detected after 300 min (i.e. 5h) of intestinal

234 digestion, showing that lipolysis was much slower for LCT than for MCT (Fig. 3B). In fact,

235 lipolysis of LCT was relatively fast during the first 30 minutes of digestion but was greatly

236 slowed down afterwards, resulting in a two-stage curve typical of most in vitro lipolysis

237 studies on submicron emulsions made of long-chain triacylglycerols [4-5, 14].

238 Higher rates of lipolysis of MCT compared to LCT have been reported in many studies using

239 pure MCT and LCT emulsions or MCT/LCT mixed emulsions as in the present study [2-5].

240 This is generally attributed to the higher water solubility of medium-chain FFAs than long-

241 chain FFAs. Indeed, the low water solubility of long-chain FFAs would lead to their

242 accumulation at the interface that would, in turn, inhibit the lipase activity by steric hindrance

243 until they are removed by bile salts or by forming soap with calcium ions [26]. In contrast, the

244 higher water solubility of medium-chain FFAs would facilitate their release from the interface,

245 and hence promote further hydrolysis of triacylglycerols at the droplet surface. According to 
246 the composition of our emulsion, other factors may also have contributed to the higher rate of 247 MCT hydrolysis. Indeed, it has been reported that triacylglycerols containing 248 docosahexaenoic acid are more resistant to pancreatic lipase [7], possibly because of an 249 inhibitory effect induced by the presence of a double bond near the carboxyl group. For

250 MCT/LCT mixed emulsions, it has also been shown that MCT hydrolysis can be promoted to 251 the detriment of that of LCT [2] because of a preferential location, or turnover, of MCT at the 252 droplet interface [2-3]. It is therefore likely that different mechanisms have contributed to the 253 marked difference we observed in the lipolysis kinetics of MCT and LCT.

254

\section{$255 \quad 4.2$ Evolution of the droplet size}

256 The evolution of the particle size distribution (measured without deflocculating agent) 257 stemming from one in vitro digestion experiment is shown in Fig. 4A. The native emulsion 258 (NE) presented a monomodal distribution with a mean $d_{32}$ of $0.26 \mu \mathrm{m}$. The mean size 259 increased considerably during the gastric phase since the measured $d_{32}$ was $3.00 \pm 0.46 \mu \mathrm{m}$ 260 after 60 min of contact with the SGF (G60). However, the size distribution and the measured $261 d_{32}$ returned close to their original values after dilution of the same sample in a $1 \%$ SDS 262 solution (not shown) or after addition of a SIF with no pancreatin or lipase (I0). This 263 demonstrates that the increase of the mean diameter during the gastric phase was caused by 264 droplet flocculation, and that the subsequent addition of bile led to a deflocculation of these 265 droplet aggregates. During the intestinal phase, the droplet size distribution remained similar 266 during the first 15 min (I15). It was suddenly shifted toward considerably larger diameters at 267 about $\mathrm{t}=30 \mathrm{~min}($ mean volume diameter of about $9 \mu \mathrm{m})$ and remained relatively stable until 268 the end of the experiment (I30 to I300), with a good repeatability of the surface weighted 269 diameters over the three replicates (Fig. 4B). 
270 According to the demonstrated tendency of bile to deflocculate the droplet aggregates formed

271 during the gastric phase, the increase of the particle size during the intestinal phase most 272 assuredly resulted from a coalescence of the oil droplets. This conclusion is moreover

273 consistent with previous studies showing that whey protein stabilized submicron emulsions 274 undergo coalescence during in vitro intestinal digestion [11, 13, 19-20]. We may even

275 highlight that the evolution of the size distributions we measured is highly similar to previous 276 results obtained during the digestion of a whey protein stabilized soya oil emulsion with an 277 initial $d_{32}$ of $0.37 \mu \mathrm{m}$, and for which an intense coalescence was observed by confocal 278 microscopy after 10 to 30 min of intestinal digestion [19]. Hence, even if flocculation cannot 279 be totally excluded from our own set of data, we will only refer to the term of coalescence in 280 the rest of this article.

281 We may also highlight the remarkable simultaneity of the increase of the droplet size (Fig. 4B) 282 and of the decrease of the lipolysis rate (LCT in Fig. 3B). Although the authors did not point 283 out this particular aspect, it seems that droplet coalescence during the intestinal phase was 284 also concomitant with a decrease of the lipolysis rate in the recent study of $\mathrm{Li}$ and coworkers 285 [19]. It is indeed well known that the rate of lipolysis decreases with decreasing surface area, 286 and hence with increasing droplet size $[3,14,16,22,27]$. We can therefore wonder how 287 much of the decrease of the LCT lipolysis kinetics at about $\mathrm{t}=30 \mathrm{~min}($ Fig. 3B$)$ was induced 288 by droplet coalescence. This was further explored using a modeling approach.

$290 \quad 4.3$ Modeling results

291 We remind that MCT was fully hydrolyzed in less than 15 min so that only LCT lipolysis was 292 considered for the modeling. The results obtained with three different mathematical models 293 are presented in Fig 5A. First, the dotted line represents our model version that assumes a 294 constant number of droplets of identical diameter (assumption A3a), and which decreases in 
295 size upon hydrolysis of the TAG they contain (Fig. 5B). The fit was very bad because the

296 assumed mechanisms could not reproduce the strong decrease of the reaction kinetics at about $29730 \mathrm{~min}$.

298 Second, the dashed line represents the model proposed by Li and McClements [5]. This model 299 is similar to the previous one but further assumes that a fraction of the TAG can remain 300 undigested. It provided a very good fit of our experimental data $\left(R^{2}=0.9888\right)$ and led to a 301 lipolysis extent of $77.3 \pm 2.6 \%$ and a surface rate constant $\left(k_{h}\right)$ of $9.4 \pm 1.5 \mu \mathrm{mol} \cdot \mathrm{m}^{-2} \cdot \mathrm{min}^{-1}$, 302 which is rather close to the $13.8 \mu \mathrm{mol} \cdot \mathrm{m}^{-2} \cdot \mathrm{min}^{-1}$ reported for a corn oil emulsion in [5]. As 303 noticed by the authors, these rate constants should nevertheless be considered as upper values 304 whenever droplet flocculation or coalescence takes place because, in such cases, the model 305 would not adequately simulate the evolution of the available interfacial area. This is also why 306 the mean diameter simulated with this model decreased from 350 to $213 \mathrm{~nm}$ (Fig. 5B), a trend 307 that is not consistent with our experimental results (Fig. 4).

308 Finally, the solid line represents our model version that accounts for the experimentally 309 measured $d_{32}($ Fig. 5A and $\mathbf{B}$ ). This model also reproduced the experimental data very well $310\left(\mathrm{R}^{2}=0.9882\right)$. The underlying interpretation is however entirely different since the marked 311 slowdown of the reaction kinetics around $30 \mathrm{~min}$ is here fully explained by the decrease of the 312 interfacial area caused by droplet coalescence (i.e. no upper fraction of the digested lipids is 313 assumed here). The only unknown parameter in this model is the surface reaction rate $\left(k_{h}\right)$ 314 that was estimated to be $2.4 \pm 0.1 \mu \mathrm{mol} \cdot \mathrm{m}^{-2} \cdot \mathrm{min}^{-1}$.

315 To support the above considerations, the interfacial surface area was calculated for both our 316 model accounting for coalescence and the model of Li and McClements (Fig. 6). According 317 to the model of $\mathrm{Li}$ and McClements, the droplet size reduces upon TAG hydrolysis, leading to 318 a corresponding decrease of the surface area. The lipolysis nevertheless plateaued (Fig. 5A) 319 despite a large remaining droplet surface area. This suggests that the interface was no more 
320 available for the enzymatic action, or in other words, that the reaction was inhibited by a

321 mechanism such as an accumulation of the reaction products at the droplet surface. According

322 to our model however, the slowdown of lipolysis was clearly, and solely, related to the sharp

323 decrease of the interfacial area induced by droplet coalescence. At $300 \mathrm{~min}$, the available

324 interfacial area was low but nonzero, in agreement with the fact that lipolysis still proceeded

325 slowly. Note also that the reaction rate constants estimated with both models reflect these

326 differences of simulated surface area since they are expressed per unit of interfacial area. This

327 is indeed why the value recovered using our model (assumption A3b) is about 4 times smaller

328 than the value estimated with the model of $\mathrm{Li}$ and McClements $\left(2.4\right.$ and $9.4 \mu \mathrm{mol} \cdot \mathrm{m}^{-2} \cdot \mathrm{min}^{-1}$,

329 respectively).

330 Taking into account the evolution of the experimentally measured droplet sizes, the

331 mathematical model allowed a good prediction of the intestinal lipolysis kinetics. The

332 decrease of the interfacial area was thus the major reason for the slowdown of the reaction

333 rate after about $30 \mathrm{~min}$. We may even highlight that our model slightly overestimates the last

334 experimental point of the kinetics at $t=300 \mathrm{~min}$ (Fig. 5A). Thus, our results are still

335 compatible with an inhibition of the enzymatic reaction at the interface, but as a second order

336 factor.

337 On the one hand, our results confirm that the kinetics of lipolysis are essentially proportional 338 to the interfacial area [22]. On the other hand, they may also offer a complementary 339 explanation for the strong decreases of the lipolysis kinetics that are frequently observed after 340 few minutes of intestinal in vitro digestions. Indeed, such slowdowns are often attributed to an 341 inhibition induced by the reaction products that accumulate at the interface, with no or little 342 attention given to a possible coalescence or flocculation of the emulsion droplets. This is most 343 probably because such phenomena have not been expected to occur in the conditions 344 encountered in the intestinal phase because of high concentrations of bile salts and no 
345 macroscopic visual evidences. Nevertheless, several recent studies have shown that droplet

346 flocculation and coalescence might in fact be encountered during the intestinal phase of in 347 vitro experiments $[11,13,17,19-20]$, similarly to what has now been established for the 348 gastric phase [14, 16-17].

349 The exact cause for the occurrence of coalescence still remains to be studied because the 350 nature of the interfacial layer is continuously evolving, especially in the case of protein351 stabilized emulsion. The TAG composition of the droplets is evolving in the course of the 352 reaction [28] and a competitive adsorption process takes place at the interface between 353 emulsifier molecules, enzymes, bile salts and the products of the lipolysis reactions. It has 354 been reported that hydrolysis of the proteins adsorbed at the interface can weaken the droplet 355 repulsion forces and favor droplet flocculation or coalescence [19, 25], possibly explaining 356 why protein stabilized emulsions seem more sensitive to these phenomena [27]. Droplet 357 coalescence during digestion has also been reported to be promoted by the accumulation of 358 monoacylglycerols and fatty acids at the interface $[16,28]$, that is, by the same mechanism as 359 that usually put forward to support an inhibition of the enzymatic reaction. More studies are 360 therefore needed to determine the frequency of droplet coalescence during in vitro intestinal 361 digestions and to better understand its consequences on the lipolysis kinetics.

\section{Conclusion}

364 Our study confirms previously reported results showing that the kinetics of lipolysis is much 365 faster for MCT than for LCT when they are mixed together in the same emulsion. In 366 agreement with recent studies, it also confirms that whey protein stabilized submicron 367 emulsions are prone to coalescence during the intestinal phase of in vitro digestions. 368 Moreover, by accounting for the experimentally measured droplet distributions in a modeling 369 approach, we were able to adequately reproduce the two-stage lipolysis curve recovered for 
370 LCT with an initial fast reaction rate that markedly slowed down after about $30 \mathrm{~min}$. These

371 modeling results demonstrate that droplet coalescence had a considerable impact on the

372 lipolysis kinetics of the remaining LCT by causing a sharp reduction of the interfacial area

373 available for the adsorption of pancreatic lipase-colipase. Contrarily to what is generally

374 postulated for intestinal lipid digestion, our findings suggest that inhibition of the enzymatic

375 reaction might not always be the key mechanism explaining why in vitro lipolysis kinetics of 376 emulsified lipids often plateaus before the reaction is completed.

\section{Acknowledgements}

379 The authors thank Valérie Beaumal (UR1268 BIA) for the preparation of the freeze-dried 380 emulsion and Claude Genot and Sebastien Marze for helpful remarks and discussions. This 381 work was supported by the Institut National de la Recherche Agronomique and the Institut 382 Carnot QUALIMENT (France). The authors are involved in the Food and Agriculture COST 383 (European Cooperation in Science and Technology) Action FA1005 'Improving health 384 properties of food by sharing our knowledge on the digestive process (INFOGEST)', 385 http://www.cost-infogest.eu 
386

387 1. Armand, M., Lipases and lipolysis in the human digestive tract: where do we stand?

\section{References} Current Opinion in Clinical Nutrition and Metabolic Care, 2007. 10(2): p. 156-164.

389

2. Deckelbaum, R.J., et al., Medium-chain versus long-chain triacylglycerol emulsion hydrolysis by lipoprotein-lipase and hepatic lipase - Implications for the mechanisms of lipase action. Biochemistry, 1990. 29(5): p. 1136-1142.

3. Armand, M., et al., Effects of droplet size, triacylglycerol composition, and calcium on the hydrolysis of complex emulsions by pancreatic lipase - An in vitro study. Journal of Nutritional Biochemistry, 1992. 3(7): p. 333-341.

4. Zhu, X., et al., Free fatty acid profiles of emulsified lipids during in vitro digestion with pancreatic lipase. Food Chemistry, 2013. 139(1-4): p. 398-404.

5. $\quad \mathrm{Li}, \mathrm{Y}$. and D.J. McClements, New mathematical model for interpreting $\mathrm{pH}$-stat digestion profiles: Impact of lipid droplet characteristics on in vitro digestibility. Journal of Agricultural and Food Chemistry, 2010. 58(13): p. 8085-8092.

6. Li, Y., M. Hu, and D.J. McClements, Factors affecting lipase digestibility of emulsified lipids using an in vitro digestion model: Proposal for a standardised $\mathrm{pH}$ stat method. Food Chemistry, 2011. 126(2): p. 498-505.

7. Bottino, N.R., G.A. Vandenburg, and R. Reiser, Resistance of certain long-chain polyunsaturated fatty acids of marine oils to pancreatic lipase hydrolysis. Lipids, 1967. 2(6): p. 489-93.

8. Marze, S., A. Meynier, and M. Anton, In vitro digestion of fish oils rich in n-3 polyunsaturated fatty acids studied in emulsion and at the oil-water interface. Food \& Function, 2013. 4(2): p. 231-239. 
409 9. Armand, M., et al., Emulsion and absorption of lipids: The importance of

410

411

412

413

414

415

416

417

418

419

420

421

422

423

424

425

426

427

428

429

430

431 17. Day, L., et al., Tailoring the digestion of structured emulsions using mixed

432

physicochemical properties. Ocl-Oleagineux Corps Gras Lipides, 1997. 4(3): p. 178-

185.

10. Borel, P., et al., Hydrolysis of emulsions with different triglycerides and droplet sizes by gastric lipase in vitro - Effect on pancreatic lipase activity Journal of Nutritional Biochemistry, 1994. 5(3): p. 124-133.

11. Mun, S., E.A. Decker, and D.J. McClements, Influence of emulsifier type on in vitro digestibility of lipid droplets by pancreatic lipase. Food Research International, 2007. 40(6): p. 770-781.

12. Hur, S.J., E.A. Decker, and D.J. McClements, Influence of initial emulsifier type on microstructural changes occurring in emulsified lipids during in vitro digestion. Food Chemistry, 2009. 114(1): p. 253-262.

13. Singh, H., A.Q. Ye, and D. Horne, Structuring food emulsions in the gastrointestinal tract to modify lipid digestion. Progress in Lipid Research, 2009. 48(2): p. 92-100.

14. Golding, M., et al., Impact of gastric structuring on the lipolysis of emulsified lipids. Soft Matter, 2011. 7(7): p. 3513-3523.

15. Marciani, L., et al., Enhancement of intragastric acid stability of a fat emulsion meal delays gastric emptying and increases cholecystokinin release and gallbladder contraction. American Journal of Physiology-Gastrointestinal and Liver Physiology, 2007. 292(6): p. G1607-G1613.

16. Wooster, T.J., et al., Impact of different biopolymer networks on the digestion of gastric structured emulsions. Food Hydrocolloids, 2014. 36: p. 102-114. monoglyceride-caseinate interfaces. Food Hydrocolloids, 2014. 36: p. 151-161. 
433 18. Nik, A.M., A.J. Wright, and M. Corredig, Surface adsorption alters the susceptibility

434

435

436

437

438

439

440

441

442

443

444

445

446

447

448

449

450

451

452

453

454 26. Zangenberg, N.H., et al., A dynamic in vitro lipolysis model I. Controlling the rate of

455

456

of whey proteins to pepsin-digestion. Journal of Colloid and Interface Science, 2010. 344(2): p. 372-381.

19. Li, J., et al., Physicochemical behaviour of WPI-stabilized emulsions in in vitro gastric and intestinal conditions. Colloids and Surfaces B-Biointerfaces, 2013. 111: p. 80-87.

20. Sarkar, A., D.S. Horne, and H. Singh, Pancreatin-induced coalescence of oil-in-water emulsions in an in vitro duodenal model. International Dairy Journal, 2010. 20(9): p. 589-597.

21. EFSA, Scientific opinion on dietary reference values for fats, including saturated fatty acids, polyunsaturated fatty acids, monounsaturated fatty acids, trans fatty acids, and cholesterol. EFSA Journal, 2010.8 (3): 1461.

22. Verger, R. and G.H. De Haas, Interfacial enzyme kinetics of lipolysis. Annual Review of Biophysics and BioEngineering, 1976. 5: p. 77-117.

23. Jurado, E., et al., Kinetics of the enzymatic hydrolysis of triglycerides in o/w emulsions: Study of the initial rates and the reaction time course. Biochemical Engineering Journal, 2008. 40(3): p. 473-484.

24. Bligh, E.G. and W.J. Dyer, A rapid method of total lipid extraction and purification. Canadian Journal of Biochemistry and Physiology, 1959. 37: p. 911-917.

25. Kenmogne-Domguia, H.B., et al., Gastric conditions control both the evolution of the organization of protein-stabilized emulsions and the kinetic of lipolysis during in vitro digestion. Food \& Function, 2012. 3(12): p. 1302-1309.

lipolysis by continuous addition of calcium. European Journal of Pharmaceutical Sciences, 2001. 14(2): p. 115-122. 
457 27. Singh, H. and A.Q. Ye, Structural and biochemical factors affecting the digestion of 458 protein-stabilized emulsions. Current Opinion in Colloid \& Interface Science, 2013.

459 18(4): p. 360-370.

460 28. Ye, A.Q., et al., Effect of calcium on the kinetics of free fatty acid release during in 461 vitro lipid digestion in model emulsions. Food Chemistry, 2013. 139(1-4): p. 681-688. 
1 Fig 1. Calibration curve relating the HPLC signal to the injected mass of TAG. The light

2 scattering detector responded the same way to both LCT (triangles) and MCT (diamonds).

3

4 Fig 2. HPLC chromatogram stemming from an undigested sample (circles) superimposed 5 with the results of the deconvolution process: LCT signal (dashed line), MCT signal (dotted 6 line) and their sum (solid line).

7

8 Fig 3. (A) Typical evolution of HPLC chromatograms during the intestinal phase of in vitro

9 digestion: native emulsion (dashed line) and, from top to bottom, at $\mathrm{t}=0,15,30,60,120$ and 10300 min after the SIF addition, respectively. (B) Extent of lipolysis for MCT (diamonds) and 11 LCT (triangles) during the intestinal phase. Means and standard deviations (smaller than the 12 symbol size) were calculated over 3 replicates.

14 Fig 4. (A) Typical evolution of the droplet size distributions measured without deflocculating agent during in vitro digestion. From back to front: Native emulsion (NE), samples taken at the end of the gastric phase (G60), and at $\mathrm{t}=0,15,30,60,120$ and $300 \mathrm{~min}$ (I0 to I300) after

17 the SIF addition, respectively. (B) Evolution of the $\mathrm{d}_{32}$ during the intestinal phase. Means and standard deviations (vertical bars) were calculated over 3 replicates.

Fig 5. (A) Extent of LCT lipolysis measured by HPLC (symbols) and the fits obtained with i)

21 our model that does not account for the observed coalescence (dotted line, assumption A3a), ii)

22 the model of Li and McClements [5] (dashed line), and iii) our model that accounts for 23 coalescence using the measured $d_{32}$ (solid line, assumption A3b). (B) Comparison of the 24 measured (symbols) and simulated $\mathrm{d}_{32}$ for the different models (same line coding). 
26 Fig 6. Evolution of the interfacial area during the intestinal phase according to our model that

27 accounts for coalescence using the measured $\mathrm{d}_{32}$ (solid line, assumption $\mathrm{A} 3 \mathrm{~b}$ ) and to the 28 model of Li and McClements [5] (dashed line).

29 


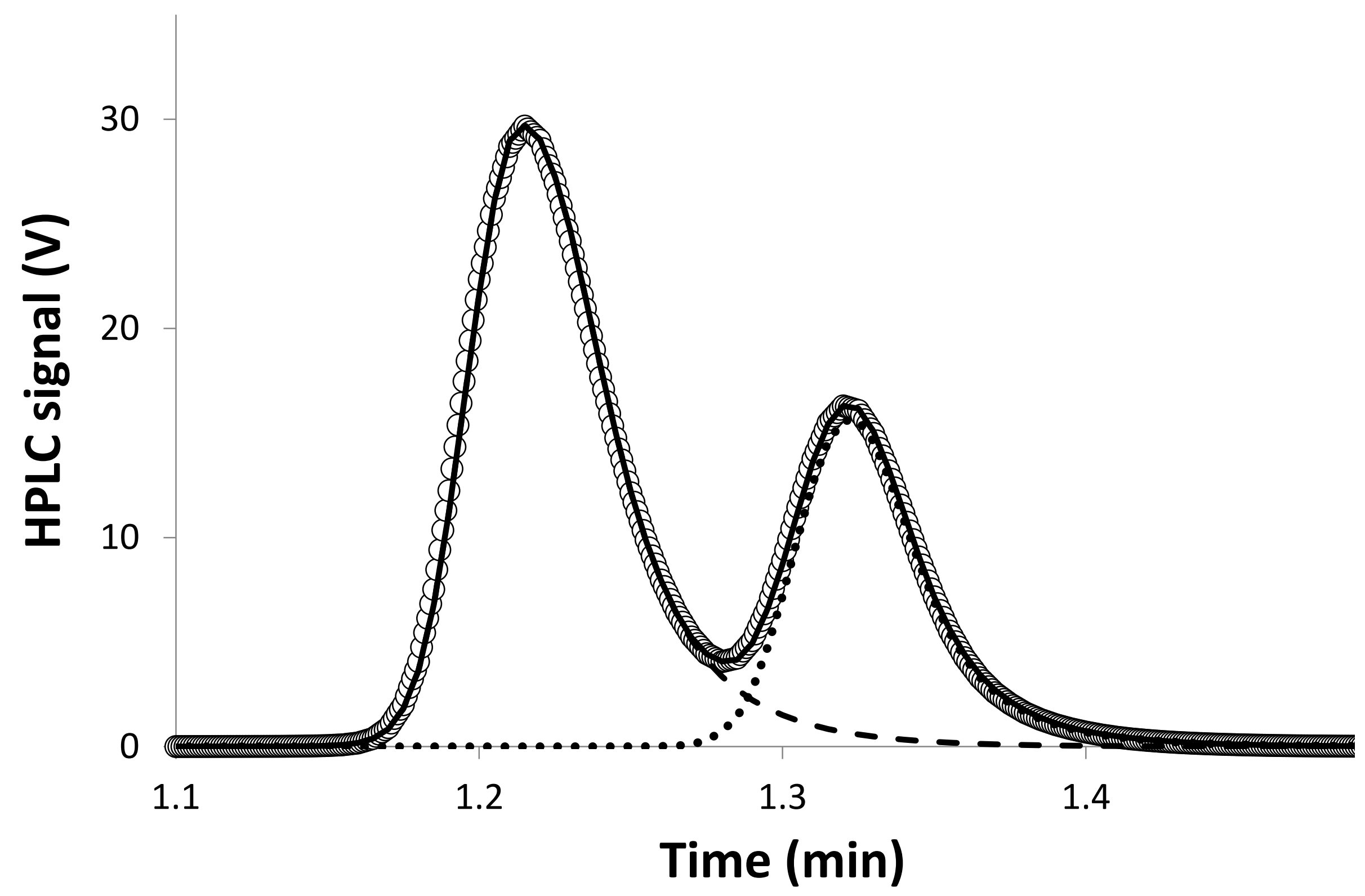




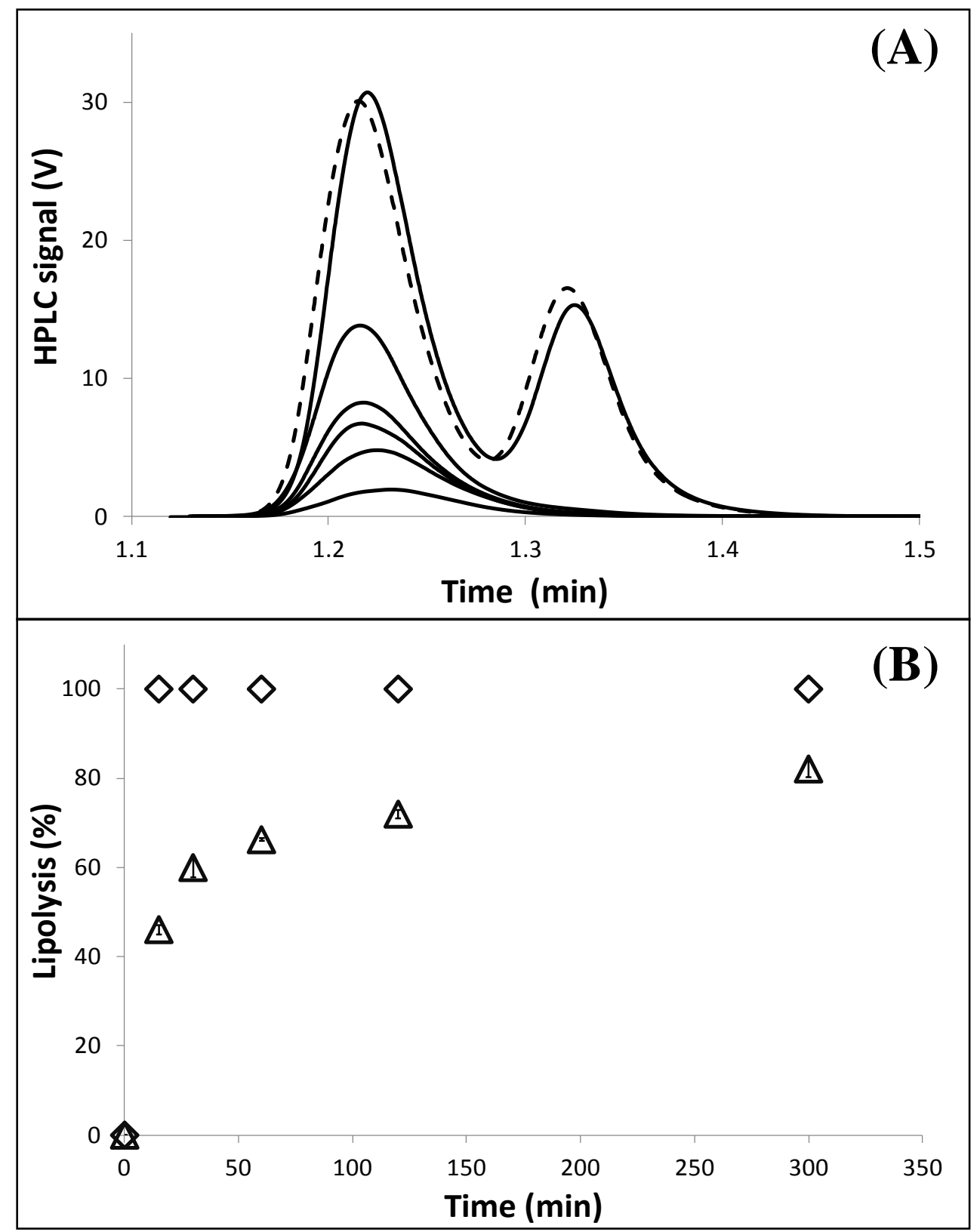




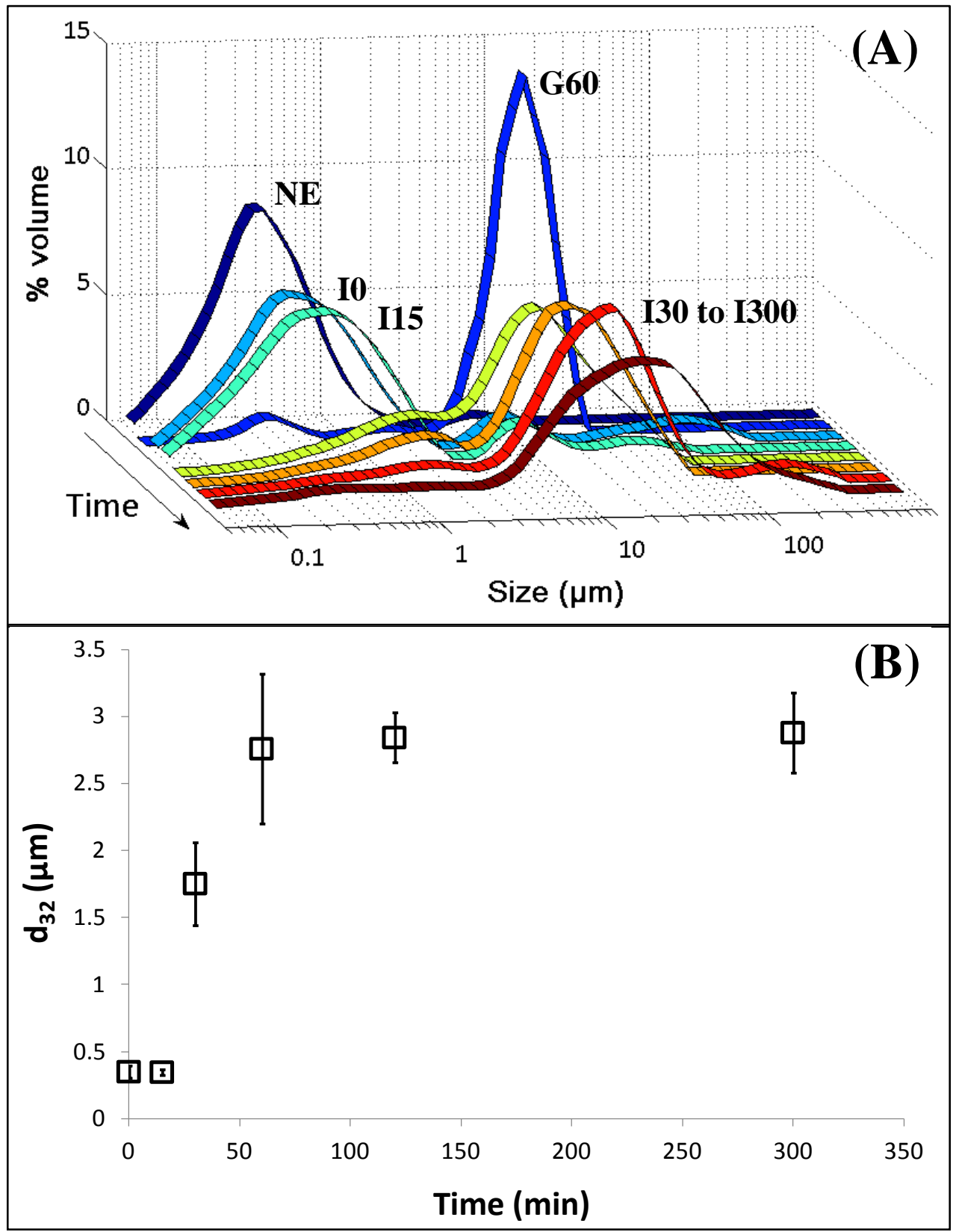




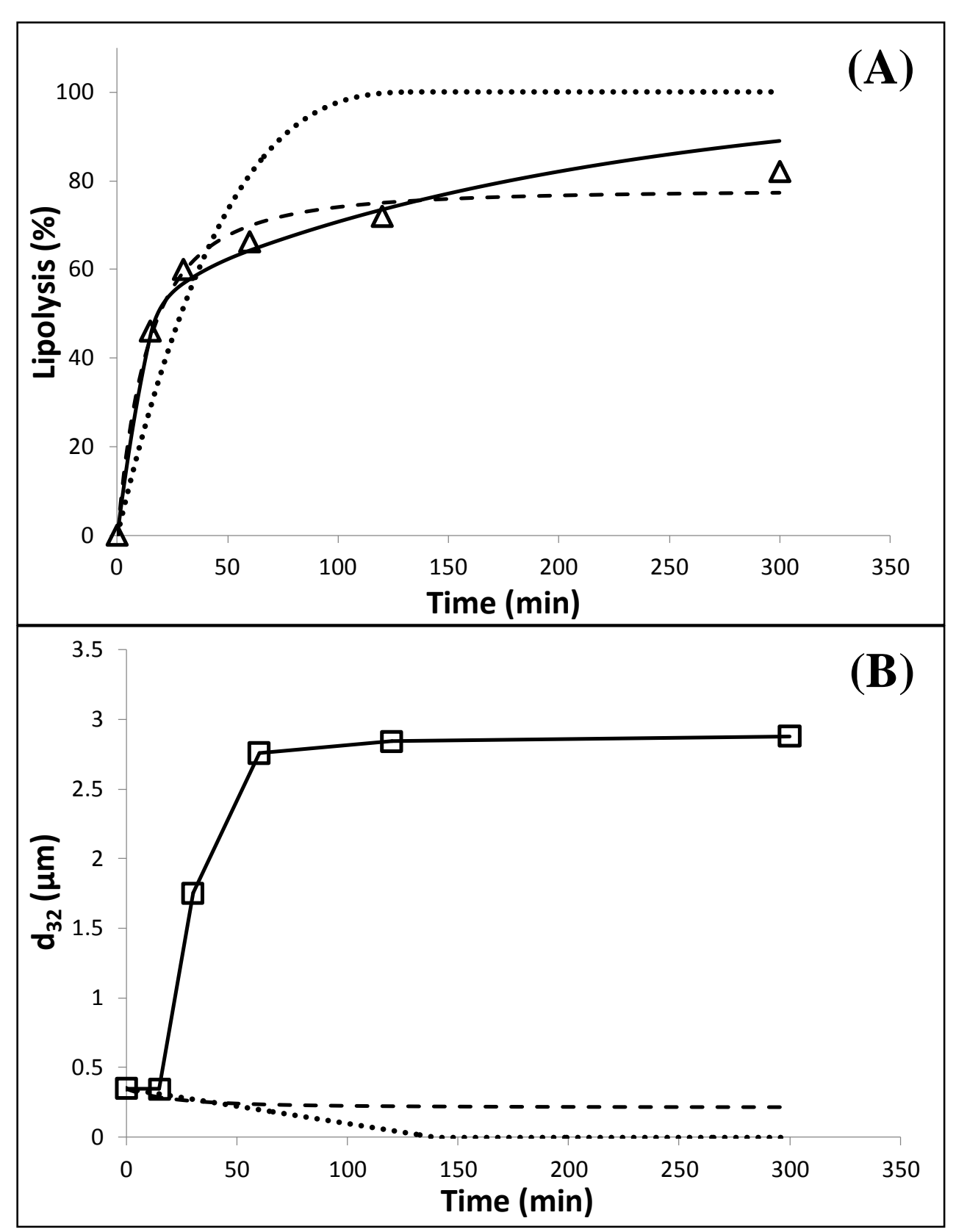




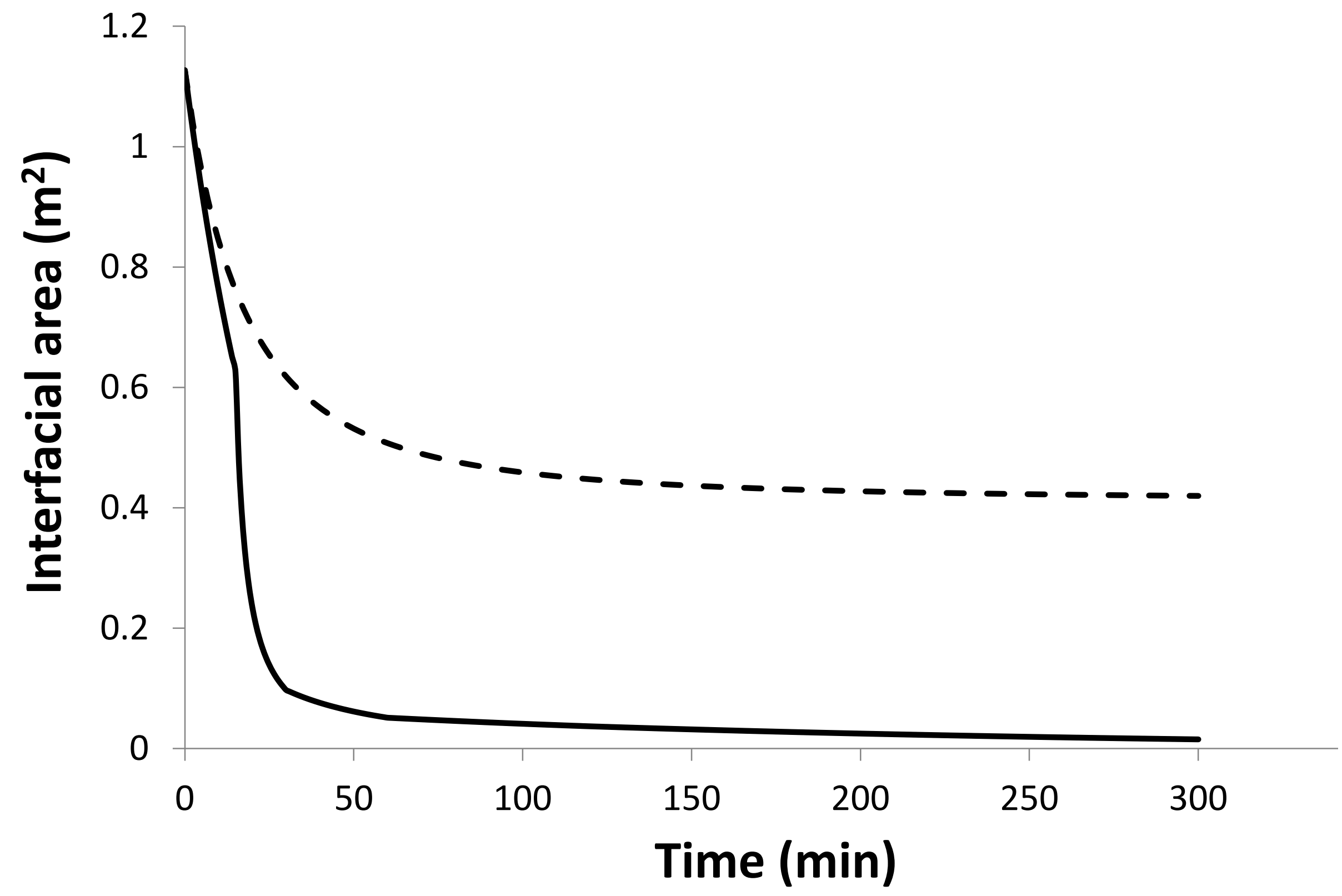

